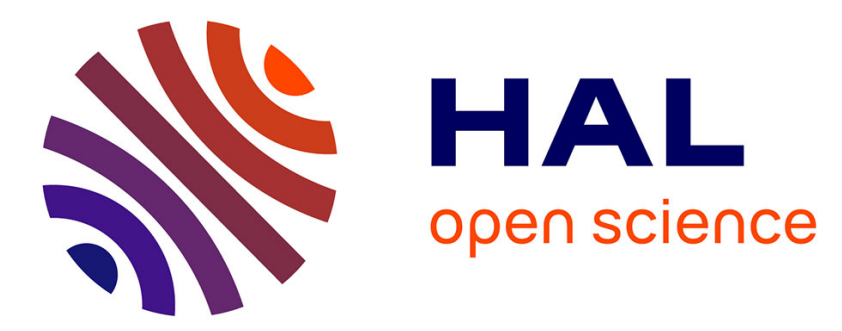

\title{
La participation à des projets artistiques, vecteur d'émancipation ? Le cas de projets de création dans des écoles montréalaises en milieu défavorisé
}

Myriam Lemonchois

\section{- To cite this version: \\ Myriam Lemonchois. La participation à des projets artistiques, vecteur d'émancipation ? Le cas de projets de création dans des écoles montréalaises en milieu défavorisé. Culture et Musées, 2015, 26, pp.159-175. 10.4000/culturemusees.386 . hal-03262739}

\section{HAL Id: hal-03262739 \\ https://hal.science/hal-03262739}

Submitted on 16 Jun 2021

HAL is a multi-disciplinary open access archive for the deposit and dissemination of scientific research documents, whether they are published or not. The documents may come from teaching and research institutions in France or abroad, or from public or private research centers.
L'archive ouverte pluridisciplinaire HAL, est destinée au dépôt et à la diffusion de documents scientifiques de niveau recherche, publiés ou non, émanant des établissements d'enseignement et de recherche français ou étrangers, des laboratoires publics ou privés. 


\section{OpenEdition} Journals

\section{Culture \& Musées}

Muséologie et recherches sur la culture

$26 \mid 2015$

Entre les murs / Hors les murs

Hors thème

\section{La participation à des projets artistiques, vecteur d'émancipation? Le cas de projets de création dans des écoles montréalaises en milieu défavorisé}

Participation in artistic projects, vector of emancipation? The case of creation's projects in Montreal' schools from unprivileged background

¿La participación en proyectos artísticos, vector de emancipación ? El caso de proyectos de creación en escuelas montrealenses en zonas desfavorecidas

MYRIAM LEMONCHOIS

p. $159-175$

https://doi.org/10.4000/culturemusees.386

\section{Résumés}

Français English Español

Nombreuses sont les publications qui énoncent les bénéfices apportés par la participation à des projets artistiques, mais bien peu ont été démontrés. Si la notion de participation renvoie à l'expérience ordinaire, d'un point de vue politique, elle n'est pas sans poser problème, et ce d'autant plus quand il s'agit de parler d'émancipation. La recherche présentée ici a pour objectif d'étudier les effets de projets artistiques sur des élèves et sur des artistes, à partir d'une définition de la notion de participation, précise et reliée aux spécificités de la création artistique. Elle s'est déroulée dans des écoles primaires et secondaires de l'île de Montréal (Canada) situées en milieu défavorisé. Les 
projets étudiés appartiennent au programme Libres comme l'art dont l'objectif est de faire participer des élèves à un projet de création artistique professionnelle sur plusieurs mois.

Many publications express benefits of participation in artistic projects, but little are proved. If the notion of « participation » is connected with the ordinary experience, from a political point of view, without raising the problem and even more when speaking about emancipation. The presented research has for objective to study the effects of artistic projects on scholars and artists, from a clear definition of « participation » focused on specificities of the artistic creation. It took place in primary and secondary schools occupying children out of an unprivileged background from Montreal's Island (Canada) from unprivileged background. The studied projects belong to the program Libres comme l'art which facilitates student's participation in a project of professional artistic creation over several months.

Muchas publicaciones describen los beneficios aportados por la participación en proyectos artísticos, pero muy pocos son aquellos que han sido demostrados. Si la noción de "participación" remite a la experiencia ordinaria, desde un punto de vista político, puede presentar ciertos problemas, y más aun cuando se habla de emancipación. La investigación que aquí se presenta tiene como objetivo estudiar los efectos de proyectos artísticos sobre estudiantes y artistas, a partir de una definición del concepto de "participación" precisa y vinculada a las especificidades de la creación artística. Se llevó a cabo en escuelas primarias y secundarias situadas en medios desfavorecidos de la Isla de Montreal (Canadá). Los proyectos estudiados pertenecen al programa Libres como el arte cuyo objetivo es hacer que los estudiantes participen en un proyecto de creación artística profesional de varios meses.

\section{Entrées d'index}

Mots-clés : intervention d'artiste, écoles primaires et secondaires, participation, intention artistique, émancipation

Keywords: visiting artist, primary and secondary schools, participation, artistic intention, emancipation

Rubriques : Varia

Palabras clave: intervención de artista, escuelas primarias y secundarias, participación, intención artística, emancipación

Notes de la rédaction

Manuscrit reçu le : 22 février 2015

Version révisée reçue le : 14 avril 2015

Article accepté pour publication le : 9 juillet 2015

\section{Texte intégral}

Il est souvent affirmé que les projets artistiques ${ }^{1}$ destinés à des élèves favorisent le développement de leurs compétences personnelles et sociales et seraient par conséquent un vecteur d'émancipation.

Cependant, peu de recherches ont démontré ces effets (Valentin, 2006 : 74) et l'argumentaire en faveur des arts repose principalement sur un argumentaire philosophique et politique (Kerlan, 2009:28). Par ailleurs, dans tous les domaines, on trouve rarement un projet qui ne soit participatif. Mais il existe aussi beaucoup de projets où l'implication demandée ne peut être confondue avec une participation active, que Joshua guetzkow (2002 : 10) oppose à la notion de «participation passive » : selon lui, une forme de participation active permet à chacun de donner du sens à ses actions en fonction de ses propres intérêts et par conséquent de faire des choix de manière constructive avec d'autres. Face à la multiplicité des projets artistiques, il est donc difficile d'en comprendre les effets sans prendre en compte la notion de participation. L'objectif de la recherche présentée dans cet article est de décrire la participation des élèves à un projet de création professionnelle avec des artistes et d'en comprendre les impacts sur les élèves et les artistes. Ses résultats amènent à s’interroger sur la participation à des activités artistiques comme projet d'émancipation, en insistant sur les notions d'égalité et de 
posture d'auteur. Avant cette présentation, nous reviendrons sur le déroulement de la recherche et les spécificités de la participation des élèves relevées.

3 La définition de la participation adoptée dans notre recherche reprend celle de Roger Hart (1992) comme processus de prise de décisions influant sur la vie d'une personne et de sa collectivité.

$4 \quad$ Cette définition de la participation d'élèves du primaire ou du secondaire est adaptée aux perspectives ouvertes par la Convention internationale sur les droits des enfants (Cide) adoptée en 1989, qui reconnaît à l'enfant des compétences suffisantes pour prendre une part active à la société même s’il est un être en développement.

5 Cette définition a été reliée aux décisions prises par l'artiste pour poser et ajuster des intentions en fonction de contraintes internes et externes, selon Éric Ilhareguy dont la recherche porte précisément sur ce travail de mise en forme par l'artiste de ses intuitions (Ilhareguy, 2008 : 69). Pour que l'activité artistique ait lieu, l'artiste doit diriger son acte volontairement, afin de maximiser les potentialités de l'œuvre et la rendre plus intelligible, signifiante et intéressante (Ilhareguy, 2008 : 72). Pour autant, le travail ne se limite pas à une résolution de problèmes qui consisterait à surmonter des contraintes de départ. Si l'artiste est conscient de réaliser ce qu'il pressent, il n'est pas toujours en mesure de pleinement déterminer les contours de son œuvre dès le début de son élaboration et doit faire appel à un « complexe intentionnel » composé d'interactions entre une intention initiale et des intentions d'ajustement (Ilhareguy, 2008 : 69). La démarche de création artistique se déclinerait selon trois étapes : poser des intentions, ajuster ces intentions et faire des choix. La participation à une telle démarche peut donc varier d'une étape à l'autre.

$6 \quad$ Toute création artistique repose sur l'appel à la « nécessité intérieure » (Kandinsky, 1989), dans lequel l'artiste puise ses mobiles et qui joue un rôle de "verdict sensible » parce qu'elle doit avoir un caractère " pénétrant et convaincant aux yeux de l'artiste » (Ilhareguy, 2008 : 129). Par ailleurs, il doit toujours avoir en tête le regard d'autrui : pour être reconnu, il doit montrer qu'il est un être unique dont l'œuvre est originale, mais aussi qu'il est capable de toucher chacun et tous en même temps (Heinich, 2005). La création artistique est une pratique qui se déroule dans un « régime de singularité » et dans un " régime de communauté $»^{2}$ (Heinich, 2005), elle possède toujours une dimension politique en tant qu'activité d'un individu ou d'un groupe d'individus ayant des conséquences sur la société. Ce qui ne signifie pas pour autant que toute création artistique relève d'un engagement politique au sens strict du terme. À partir de la notion d'auteur comme aptitude du sujet à produire du social, par sa position de sujet et par sa force critique (Bertucci, 2007 : 14-16), on peut se demander quel type de participation à un projet de création artistique permet à l'enfant d'approcher une posture d'auteur. Pour certains, la réponse à cette question peut sembler évidente parce que des philosophes ont longuement insisté sur les liens intrinsèques entre art et émancipation de l'individu. Mais elle ne l'est plus quand il s'agit de se questionner sur ce que l'on entend par « participation » et par « démarche de création artistique ».

7 D’une manière générale, la clé de la réussite de la participation des enfants est le partage de décisions avec des adultes (Cook et al., 2004 : 14-15). La même constatation est faite par les chercheurs qui ont étudié les interventions d'artistes dans des écoles : les plus signifiantes recherchent une coconstruction entre les élèves et les artistes (Pringle, 2008 : 46). Le contexte des projets avec des artistes est donc pertinent pour étudier une forme de participation dans les écoles, qui pourrait être vecteur d'émancipation des élèves.

\section{Les projets Libres comme l'art et le déroulement de la recherche}


Les projets Libres comme l'art permettent à des élèves de l'île de Montréal (Canada) de prendre part à un projet de création artistique professionnelle pendant plusieurs mois. Initiés par le Conseil des arts de Montréal, Le Conseil des élus de Montréal et l'École montréalaise pour tous, ils sont proposés aux écoles dont les indices de défavorisation sont élevés. Ces indices sont établis chaque année par le ministère de l'Éducation du Québec à l'aide de deux variables : un indice du seuil de faible revenu et un indice de milieu socioéconomique défavorisé composé de la sous-scolarisation de la mère et de l’inactivité des parents. Les enquêtes de participation culturelle montrent que la défavorisation correspond le plus souvent à un faible taux de fréquentation des arts et des lieux culturels (Pronovost, 2002 : 34). Les projets Libres comme l'art s'adressent donc à des élèves éloignés des milieux artistiques.

9 La recherche a adopté une approche qualitative en utilisant deux outils de collecte de données : des observations et des entretiens avec des artistes, des enseignants et des élèves. L'analyse de contenu a consisté en une analyse thématique selon les notions développées dans le cadre de référence (participation, démarche de création). Des catégories ont ensuite été créées pour articuler toutes les données recueillies. L'analyse a été transversale, croisant données de terrain et points de vue théoriques.

La recherche a commencé en 2012 et le recueil de données s'est achevé en 2014. L'ensemble des disciplines artistiques admissibles au programme Libres comme l'art ${ }^{3}$ a pu être couvert lors de la cueillette des données. Douze entretiens d'une à deux heures ont été réalisés avec des artistes. Des problèmes de demande d'autorisation pour enquêter dans les écoles ont empêché de rejoindre l'ensemble des élèves et des enseignants ayant participé aux projets : seulement six entretiens d'une heure avec des groupes de trois ou quatre élèves ont été réalisés et six entretiens d'une heure avec des enseignants, ainsi que neuf heures d'observations et trois entretiens avec des conseillères pédagogiques. Cinq des six enseignants interviewés sont des spécialistes en enseignement des arts, qui peuvent intervenir aussi bien en primaire qu'en secondaire, et quatre d'entre eux sont des hommes. L'autre enseignant est une femme spécialiste du français dans des écoles secondaires, très familière des pratiques artistiques. Tous les entretiens de groupe avec des élèves ont été effectués avec des enfants dont les classes avaient été observées : un avec des élèves de 2e année du primaire (7-8 ans) et cinq avec des élèves de 5e année du secondaire (16-17 ans). Quant aux trois conseillères pédagogiques, elles sont spécialistes d'un des domaines artistiques au programme et peuvent intervenir auprès d'enseignants du primaire et d'enseignants spécialistes en art. Les groupes de participants à la recherche sont donc relativement homogènes : majoritairement des élèves de 16-17 ans et des enseignants spécialistes de l'enseignement des arts.

\section{La participation des élèves aux projets Libres comme l'art}

11 Quel que soit le niveau des élèves, les artistes les ont impliqués dans leur processus de création : poser des intentions initiales, les ajuster et faire des choix.

\section{Poser des intentions initiales}

12 Les intentions de départ ne sont pas déterminées par les élèves étant donné qu'elles sont définies lors de la constitution du dossier de candidature des artistes. Par ailleurs, ce ne sont pas toujours les artistes qui les ont définies : ainsi, une romancière rapporte que le thème de la création littéraire lui a été imposé par l'organisme culturel. Ces intentions sont ce qu'un des artistes rencontrés appelle "des grandes lignes », un autre " une idée générale ». Mais pour que les élèves participent à la démarche de l'artiste, il doit les 
amener à se les approprier. Nous verrons un peu plus loin comment cette étape se traduit dans la relation des artistes avec les élèves.

\section{Ajuster les intentions}

Pour l'ensemble des artistes rencontrés, la participation des élèves a consisté principalement à ajuster les intentions. Pour certains, par des activités d’idéation 4 : les élèves doivent choisir parmi diverses possibilités d'ajustements lesquelles sont les plus pertinentes selon leur point de vue et celui des artistes. Par exemple, des danseurs ou des musiciens interprètent des séquences et les élèves les critiquent. Dans d'autres cas, par l'expérimentation : par exemple, des élèves du secondaire vont rechercher diverses phrases musicales qu'ils transcriront sur papier 5 et vont éventuellement jouer ensuite, pour trouver laquelle sonne le plus juste en fonction du projet. Pour les artistes, l'ajustement, que ce soit par idéation ou par expérimentation, il a la même valeur ; en revanche, pour la plupart des acteurs de l'école (enseignants ou conseillers pédagogiques), l'idéation n'est pas reconnue comme une modalité de participation à la démarche de création de l'artiste.

Pour les ajustements, un des artistes indique qu'il devait faire comprendre aux élèves que la démarche de création implique " des remises en question, des transformations ", parce que, de son côté, il passe son « temps à réaménager cela ». Dans le primaire comme dans le secondaire, les artistes ont toujours essayé d'expliquer pourquoi ces ajustements ne pouvaient être définitifs et précisé qu'ils pouvaient toujours être remis en cause jusqu'à la fin de la réalisation : "C'était un travail en progression, les jeunes nous proposaient des choses, on en discutait avec eux, et ensemble on voyait si ça pouvait fonctionner, comment on pourrait l'arranger », nous dit l'un d'eux, qui est intervenu auprès d'élèves du primaire.

\section{Faire des choix}

En classe, ce que les artistes demandent aux élèves rappelle ce que l'artiste Vassily Kandinsky nomme la « nécessité intérieure ${ }^{6}$ » : « C'est toi qui sais le mieux ce qui est bon » est une phrase souvent répétée aux élèves durant les interventions que nous avons observées au primaire et au secondaire. De cette façon, ils renvoient les élèves à leur intériorité et leur attribuent une compétence artistique : celle de pouvoir déterminer ce qui est juste en fonction du projet et de leur propre sensibilité.

Pourtant, les choix des élèves sont souvent revus par les artistes. En entretien, ces derniers nous disent qu'ils ont essayé de les respecter le plus possible tout en les adaptant en fonction de leurs critères artistiques. Les choix des élèves ne sont donc pas tous incorporés dans la réalisation finale ou sont transformés. D’une manière générale, le fait que leurs décisions ne se retrouvent pas toutes dans le produit final ne les a pas dérangés. Des élèves du primaire qui ont participé à un projet de conte musical nous disent par exemple : "S'ils le mettent là, on serait contents, parce que notre choix est mis dans le spectacle, mais si jamais ils sont pas là, [...] au moins on a pu dire notre personnalité, les choix qu'on a choisis. » Les élèves ajoutent qu'ils se sont sentis " comme des créateurs » parce qu'ils ont pu exprimer leur personnalité dans le processus de création, même si tous leurs choix n'ont pas été intégrés. Que les artistes interviennent pour transformer ou retirer les choix des élèves ne diminue pas leur participation selon les artistes et les élèves, mais pas d'après les enseignants. Nous discuterons de ces différences un peu plus loin.

\section{La relation entre artistes et élèves}


Parce qu’il était important pour les artistes d'amener les élèves à partager leur intention de départ, de manière unilatérale, tous les artistes ont insisté en entretien sur la nécessité de créer une rencontre particulière avec les élèves et sur la difficulté de le faire. Cette difficulté est reliée à ce que Philippe Perrenoud (1995) appelle le « métier d'élève » : l'élève apprend à répondre aux contraintes de l'école et de l'enseignant et à laisser « son moi à la porte de l'école », selon les propos d'un des élèves du secondaire interrogés. Pour établir une rencontre, les artistes doivent les sortir de la logique scolaire pour les faire entrer dans une logique artistique : amener l'élève « à être vraiment là et attentif » dans une rencontre suffisamment signifiante pour " apporter quelque chose aussi bien à l'élève qu'à l'artiste ». Mais elle n'est pas magique selon les artistes, elle doit se construire « en tenant compte des individualités ». Tous les enseignants pensent que les artistes auraient pu gagner du temps en expliquant davantage aux élèves ce qu'ils attendaient d'eux : selon eux, l'artiste perdait du temps pour des activités qui ne leur semblaient pas au premier abord être reliées au projet de création.

\section{Les effets artistiques sur les élèves}

Les effets dits « artistiques » sont les effets sur les apprentissages disciplinaires en art définis par le Programme de formation de l'école québécoise (PFEQ) (gouvernement du Québec, 2001), mais aussi ceux sur des apprentissages artistiques dont ce programme ne traite pas.

\section{Les effets sur les apprentissages artistiques au programme}

Dans le primaire et le secondaire, le curriculum scolaire québécois (gouvernement du Québec, 2001) définit deux compétences communes aux quatre arts (art dramatique, arts plastiques, danse et musique) enseignés dans les écoles : " créer » et " apprécier ». Du point de vue de l'ensemble des participants à la recherche, la compétence « apprécier » est la plus développée dans les projets ; la compétence « créer » l'est aussi, mais dans une moindre mesure. Selon une conseillère pédagogique, les projets Libres comme l'art développent la compétence " apprécier » des élèves, parce qu'ils apprennent à avoir " l'esprit critique, un regard critique sur son œuvre ». En effet, ils doivent évaluer des intentions en tenant compte de ce qu'ils ressentent et des attentes du public, pour ensuite les ajuster de manière adéquate. Tous les élèves rencontrés disent aussi avoir développé cette compétence. Une des élèves du secondaire qui a participé à un projet de création sonore nous dit : " Je vais continuer à écouter les musiques que j'aime, mais je vais essayer d'écouter vraiment en arrière pour comprendre comment ils ont modifié, comment ils les ont apprêtées. Je vais être plus curieuse au niveau musical. Pas au point de créer des chansons, mais peut-être pour élargir mes connaissances à travers la musique et la musique que j'écoute quotidiennement. » Un autre élève du secondaire dit : " $\mathrm{Au}$ moins ça m'a permis de voir plus comment la musique elle est faite. Parce que dans la musique y a plusieurs choses, si on aime vraiment la voix de la chanteuse, par exemple, tu peux te rendre compte qu'y a vraiment plusieurs instruments de musique. Mais la plupart du temps quand on écoute la musique c'est la chanteuse [...]. Tandis que là, je vais vraiment écouter les instruments et tout ça. » Si le projet n'a pas pour effet de modifier les choix artistiques des élèves, il change leur manière de les apprécier : ils ont appris à écouter ou voir des œuvres en cherchant à comprendre comment elles sont fabriquées et à prendre en compte des éléments qu'ils négligeaient auparavant. Il semble pertinent de dire que les projets changent le rapport à l'art des élèves : il ne s'agit plus seulement de consommer une œuvre juste pour le plaisir, mais de le faire avec le désir de comprendre 
comment elle a été élaborée, c'est-à-dire de reconstruire le processus de création de l'artiste.

20 La plupart des artistes ont l'impression d'avoir aidé les élèves à développer la compétence " créer », mais pas tous ses aspects par manque de temps, et regrettent de ne pas être allés « plus en profondeur, plus en qualité dans le travail de création », vers « un certain professionnalisme ». Selon eux, cette exigence de professionnalité est au cœur de leur travail. C'est pourquoi ils parlent le plus souvent d'« initiation à la création ».

21 La démarche de création apportée par les artistes diffère de ce que les élèves abordent durant les cours d'art. Selon une conseillère pédagogique, dans les projets Libres comme l'art, « la démarche artistique n'est pas nécessairement aussi explicite. On sait un peu avec quoi on va travailler, sur quel thème, on sait l'intention de l'artiste, mais on ne sait pas nécessairement quel résultat ça va donner. Il y a beaucoup plus d'incertitudes dans la démarche artistique que dans ce qu'ils vivent en classe ». La démarche de création dans les projets était plus difficile pour les élèves : en classe, l'enseignant donne des critères d'évaluation de leurs travaux stabilisés et formulés a priori, tandis que l'œuvre réalisée avec les artistes devait s'inscrire dans le monde de l'art, où les critères de discrimination de ce qui est appelé « art » font l'objet d'âpres discussions.

\section{Les autres apprentissages artistiques des élèves}

Les effets artistiques qui ne sont pas énoncés tels quels dans le curriculum des écoles primaires et secondaires du Québec sont divers : questionner la figure de l'artiste ; se confronter à l'exigence artistique ; vivre une expérience esthétique et être initié à la création contemporaine.

Les artistes ont souvent commencé le projet en essayant de « démystifier les artistes et le processus de création parce que souvent les médias montrent seulement la réussite des artistes en donnant l'impression que créer c'est facile », nous dit l'un des artistes. Pour un autre, il s'agissait de montrer aux élèves que « ce sont des choses qui s'apprennent » et qu' « il n'y a rien de magique dans cela ». Changer les représentations sur l'artiste ne semble pas être un effet spécifique aux projets Libres comme l'art : des élèves du secondaire ont dit qu'ils savaient déjà que les artistes étaient " des gens comme nous autres » parce qu'ils avaient déjà eu l'opportunité d'en rencontrer dans d'autres projets.

Par ailleurs, parce que le projet doit aboutir à une œuvre professionnelle7, les artistes disent avoir eu des standards de qualité élevés. Ils ont tous décrit cette exigence comme un élément propre aux projets Libres comme l'art : dans les autres projets qu'ils développent dans des écoles, elle n'est pas là parce que ces projets sont de courte durée ou parce que leur finalité est d'aider les élèves à élaborer leur propre projet de création. L'évaluation d'une œuvre par un large public est différente de celle à laquelle les élèves se confrontent à l'école. En effet, si l'enseignant essaie d'être exigeant avec ses élèves, il reconnaît aussi qu'il doit parfois accepter que « le rendu n'est pas très bon, et que les élèves ont donné leur maximum », nous dit l'un des enseignants du secondaire.

Si les notions de plaisir et d'effort sont séparées à l'école, en art elles peuvent être réunies. Les élèves du primaire nous le rappellent quand ils disent : " On est très contents même si ça nous prend beaucoup d'efforts. » La recherche sur les projets Libres comme l'art semble confirmer ce que d'autres chercheurs ont observé : les interventions d'artistes permettent aux élèves de découvrir « la rigueur du travail artistique et leur propre capacité à dialoguer avec le processus de création » (Valentin, $2006: 53$ ).

Tous les artistes rencontrés associent l'expérience esthétique à l'exigence artistique. Mais les acteurs de l'école diffèrent sur ce point. Selon les artistes, l'expérience esthétique n'est présente que dans un travail d'ajustement d'intentions en fonction des critères des milieux artistiques, tandis que pour les enseignants, elle serait toujours présente à partir du moment où les élèves sont en contact avec l'art. Selon John Dewey (2010 : 298-299), l'expérience esthétique est l'expression et l'organisation de nos désirs en fonction de buts 
et de fins désirables. Qu'en est-il de cette expérience à l'école durant les activités artistiques ? C'est une question légitime non seulement dans les projets, mais aussi dans les cours d'art. La recherche ne nous permet pas de déterminer qui, des artistes ou des enseignants, a raison sur ce point, mais simplement d'affirmer que leurs points de vue diffèrent. Cependant, ce résultat de recherche amène à s’interroger sur les résultats énoncés par les travaux sur les projets artistiques dans les écoles, selon les participants interrogés, qui sont le plus souvent des enseignants.

$27 \quad$ Les projets favoriseraient le développement de nouvelles conceptions de l'art, mais de l'avis d'une conseillère pédagogique, il n'est pas toujours facile de faire évoluer les représentations des élèves. Elle cite le cas d'un projet où les élèves n'avaient invité personne à la présentation du produit fini parce que cela ne leur paraissait pas assez beau, même s'ils étaient fiers de leur travail durant le projet. Cet exemple indique que leur approche de la création contemporaine reste problématique. La recherche sur les dissonances générées par l'art contemporain montre que seule une familiarisation dans la durée à ce type d'art peut aider à résoudre ces difficultés (Emond, 2010 : 61). Même les projets d'une durée étendue sur plusieurs mois restent des projets ponctuels, qui ne peuvent avoir les mêmes effets qu'une fréquentation répétée de l'art.

\section{Les effets sur les artistes}

En entretien, tous les artistes disent que le projet Libres comme l'art ne les a pas obligés à mettre de côté leur création pour être au service de l'école. Ils ont assez peu parlé des modifications sur leurs pratiques que les projets auraient pu induire et disent ne pas avoir eu d'attentes particulières, puisque pour eux le plus important était d'être sélectionnés pour réaliser leur création dans une école. En les écoutant, on se rend compte qu'ils voulaient surtout jouer un rôle de médiateur auprès des élèves. L'un d'entre eux dit que l'objectif de ses interventions était « d'allumer ces petites flammes, d'ouvrir ses horizons, en fonction d'une autre réalité qui est la culture », un autre qu'il avait envie « de partager, de former la relève, ne serait-ce que d'ouvrir les horizons ».

Plusieurs artistes disent avoir déposé un projet, parce que leur démarche de création implique des « êtres humains » et que travailler avec des enfants ou des adolescents leur paraissait « naturel ». Par exemple, l'un des artistes dit que l'intervention prévue correspondait bien à sa manière de créer, parce que sa démarche artistique est " très inclusive, parce que les gens participent ». Pour d'autres artistes dont le public est composé d'enfants ou d'adolescents, le projet représentait une opportunité de créer en lien direct avec eux. L'une des artistes interviewées précise que « c'était bon d'avoir leur assistance » parce qu' « habituellement, je travaille seule à mon bureau ». Dans les projets, la plupart des artistes recherchent auprès des élèves un regard critique sur leur création, et pour certains, les élèves sont des " matériaux humains » de leur œuvre parce que leur démarche de création est inclusive.

En entretien, tous les artistes affirment qu'ils étaient soucieux de parvenir à créer une œuvre répondant aux critères du monde de l'art. Par conséquent, ils devaient concilier deux demandes contradictoires à première vue : ne "rien ajouter » à la création des élèves - qui n'ont pas toujours les bases pour créer - et en même temps produire une œuvre de qualité professionnelle. Ceux qui ont une démarche de création inclusive n'ont pas eu de difficulté à gérer cette contradiction : ils trouvent normal que leurs œuvres soient influencées par les élèves, car elles le sont toujours en fonction du milieu où elles s'ancrent. Ceux dont les œuvres s'adressent à un jeune public ont aimé le défi car il leur permettait d'inclure dans leurs œuvres des éléments inattendus. L'une des artistes dit par exemple : "Ils m’ont emmenée à des endroits que je n'avais pas imaginés. Cela m’a tellement surprise, je serais complètement folle de ne pas me servir de cela. » Les projets Libres comme l'art permettent aux artistes de sortir de leur routine et de leur monde pour prendre du recul sur leur œuvre en cours de réalisation. Par ailleurs, parce que ces projets 
impliquent une école, un organisme culturel, le Conseil des arts et la conférence régionale des élus de Montréal, les artistes pensent que le projet leur a permis d'accroître la visibilité de leur création auprès d'un plus large public.

Si tous les artistes reconnaissent que les élèves ont influencé leur œuvre, ils n'y accordent pas le même statut. Certains la signent avec les élèves, d'autres ne la signent pas et considèrent qu'elle a été réalisée par les élèves sous la direction de l'artiste. Tous affirment que l'œuvre réalisée durant le projet est différente de toutes les autres qu'ils ont créées parce qu'elle serait plus " kitsch ", plus " naïve »: les élèves manquent de connaissances en histoire de l'art et ont tendance à plagier d'autres œuvres sans le savoir ou à « grossir le trait ». Mais, quel que soit le statut que l'artiste accorde à cette œuvre et l'influence que l'élève lui a fait subir, tous reconnaissent que c'est une œuvre d'art, qu'elle fonctionne comme telle avec sa cohérence.

\section{Discussion des résultats}

Deux résultats de cette recherche sont liés à la valeur émancipatrice accordée habituellement aux arts : les différences entre milieu scolaire et milieu artistique concernant les effets artistiques sur les élèves et les particularités de la parti- cipation des élèves dans les projets Libres comme l'art.

\section{Les différences entre milieu scolaire et milieu artistique}

Notre recherche montre que les représentations de l'art influent sur celles que se font les artistes et les acteurs de l'école des effets des projets sur les élèves. Les acteurs de l'école ont tendance à minimiser la participation des élèves et les effets artistiques sur la compétence à créer des élèves : parce qu'ils conçoivent la création comme une production d'idées et une mise en forme matérielle de ces idées. Quant aux artistes, ils relativisent leurs propos et considèrent qu'apprendre à ajuster des intentions est un apprentissage essentiel pour savoir créer, mais que cela n'est pas suffisant, le plus important étant la confrontation à l'exigence artistique attendue par le monde de l'art. Du côté de l'appréciation, les acteurs de l'école s'accordent pour dire que les projets permettent de développer cette compétence, puisqu'ils développent l'acquisition de nouvelles connaissances artistiques. Quant aux artistes, ils constatent surtout l'éloignement des élèves du monde des arts et les limites de leur capacité à apprécier des œuvres qui sortent de leurs repères culturels habituels.

Plusieurs recherches montrent qu'il n'existe aucune preuve de transfert en classe des apprentissages acquis dans des projets artistiques (Valentin, 2006 : 74). Par ailleurs, pour qu'il y ait transfert, l'enseignant doit explicitement montrer aux élèves que des éléments communs existent entre ce qu'ils font en classe et dans les projets. Or, nous avons constaté que la démarche de création adoptée par les artistes diffère de celle que les enseignants proposent aux élèves. La prudence est donc de mise quand il s'agit de mesurer le transfert des apprentissages d'un contexte à un autre, surtout quand la conception de la création des artistes est éloignée de celle des acteurs de l'école.

\section{Les particularités de la participation des élèves}

Dans les projets Libres comme l'art, même si la participation des élèves a lieu dans un contexte scolaire en respectant ses contraintes, elle diffère sur de nombreux points de celle demandée aux élèves en classe. Lors de la mise à l'épreuve des intentions artistiques, ils 
ont pu prendre des décisions quant aux ajustements nécessaires et se sont sentis reconnus comme compétents et dans une relation égalitaire avec l'artiste. Nous retrouvons ici ce que d'autres chercheurs ont pu observer dans les interventions d'artistes en milieu scolaire : une relation particulière où l'artiste se place « à côté » des élèves (« alongside ») (Pringle, 2008 : 44), où la différence entre enfant et adulte s'efface au profit d'une certaine égalité (Kerlan, 2011). Ce rapport égalitaire entre adultes et enfants n'est pas sans rappeler le projet d'émancipation décrit par Jacques Rancière $(1987,2009)$.

Prendre pour point de départ l'égalité, c'est partir non pas de ce que l'élève ignore, mais de ce qu'il sait, et vérifier s'il a usé de son intelligence (Rancière, $1987: 51$ ). Nous avons constaté que les artistes ont demandé aux élèves de justifier leurs choix, de rendre compte de leur volonté de donner du sens, en montrant que leur nécessité intérieure a bien été sollicitée. Les artistes ont donc joué un rôle identique à celui du " maître ignorant » décrit par J. Rancière (1987), en s'assurant que l'élève a cherché et en jugeant de sa capacité à vérifier par lui-même ce qu'il a trouvé. Selon J. Rancière (1987), la leçon émancipatrice de l'artiste est d'effectuer une double démarche : ressentir et faire partager ce qu'il ressent. Cela ne signifie pas copier l'œuvre d'un artiste, mais adopter une attention et une attitude de recherche propres à l'art pour dire et faire partager son rapport à soi, aux autres et au monde dans des matériaux a priori arbitraires et dont il faut forcer la résistance pour pouvoir se dire (Rancière, 1987 : 120). Pour donner cette leçon d'émancipation intellectuelle, J. Rancière (1987 : 120) ajoute que " l'artiste a besoin de l'égalité comme l'explicateur a besoin de l'inégalité ». Attribuer une valeur émancipatrice à la participation d'élèves à des projets artistiques dépendrait par conséquent d'un rapport égalitaire entre l'artiste et les élèves, dans lequel ces derniers apprennent à faire l'effort de partager ce qu'ils ressentent au plus grand nombre, comme dans le cas de la production d'une ouvre d'art professionnelle.

\section{Conclusion}

La recherche nous a conduit à constater combien les représentations de la démarche de création artistique peuvent amener à valoriser ou à nier les effets des projets artistiques. Par ailleurs, le mode de participation des élèves dans les projets artistiques semble essentiel aux yeux des artistes, tandis que les acteurs de l'école n'y accordent pas la même importance. En effet, pour les artistes, le fait que les élèves participent de manière active signifie qu'ils ajustent des intentions et font des choix au même titre que les artistes, et cela dans une expérience esthétique associée à l'exigence artistique propre au monde de l'art. Tandis que les enseignants appellent «participation active » le fait que les élèves manipulent des matériaux dans une expérience esthétique qui serait toujours présente dans le contact avec des artistes ou des œuvres. Notre recherche a adopté une approche descriptive, qui ne nous permet pas de vérifier et de mesurer les effets de cette participation sur les apprentissages des élèves. Nous ne pouvons qu'inviter les recherches à venir à en tenir compte, car la définition accordée aux arts a un impact sur la conduite des projets, mais aussi sur les représentations de leurs effets.

Par ailleurs, la recherche a permis d'observer une forme de participation dans un contexte scolaire qui implique une reconnaissance de la compétence des élèves comme prémisse, alors qu'habituellement la compétence de l'élève y est reconnue comme finalité. Plusieurs questions émergent : peut-on penser l'enseignement dans les écoles en fonction de cette forme de participation, c'est-à-dire introduire plus d'égalité dans un rapport pédagogique qui repose habituellement sur une inégalité de savoirs et de pouvoir entre l'enseignant et l'élève, en particulier dans les enseignements artistiques où elle semble essentielle ? L'expérience d'une égalité des sujets étant ponctuelle, que devient la valeur émancipatrice de ces projets à plus ou moins long terme? 


\section{Bibliographie}

Bertucci (Marie-Madeleine). 2007. « La notion de sujet », in Le Français aujourd’hui, 157, p. 11-18. DOI : 10.3917/lfa.157.0011

Bonnardel (Nathalie). 2006. Créativité et conception : Approches cognitives et ergonomiques. Marseille : Solal.

Brougère (Gilles). 2012. " Participation, learning and intercultural expérience ", p. 180-196 in Participation, Facilitation and Mediation / sous la direction de Baraldi (Claudio) \& Ieverse (Vittorio). Londres : Routledge.

Cook (Philip), Blanchet-Cohen (Natasha) \& Hart (Stuart). 2004. Children as Partners : Child Participation Promoting Social Change. Victoria, Canada : IICRD.

Dewey (John). 2010[1934]. L'Art comme expérience. Paris : Gallimard.

Émond (Anne-Marie). 2010. " Positive responses of adult visitors to art in a museum context ", p. 43-62 in Essays on Aesthetic Education for the 21st Century / sous la direction de Constantino (Tracy) \& White (Boyd). Rotterdam : Sense Publishers.

Gouvernement du Québec. 2001. Programme de formation de l'école québécoise. Québec : ministère de l'Éducation.

Guetzkow (Joshua). 2002. " How the arts impact communities : An introduction to the literature on arts impact studies ». Working Paper Series, Princeton University, 20, p. 1-26.

Hart (Roger). 1992. Children's Participation : From Tokenism to Citizenship. Florence : International Child Development Center.

Heinich (Nathalie). 2005. L'Élite artiste : Excellence et singularité en régime démocratique. Paris : Gallimard.

Ilhareguy (Éric). 2008. Mise à l'épreuve d'intentions artistiques : Étude de la dynamique interne des processus de créativité. Thèse de doctorat non publiée, université de Montréal, Québec.

Kandinsky (Vassily). 1989. Du spirituel dans l'art, et dans la peinture en particulier. Paris : Denoël.

Kerlan (Alain). 2009. "L'entrée éducative : La culture comme expérience et rencontre ». Champs culturels, 22, p. 26-31.

Kerlan (Alain). 2011. "L'atelier de l'artiste, laboratoire démocratique d'une nouvelle normativité ? ». Sens Public. Publication en ligne : http://www.sens-public.org/spip.php?article894. Consulté le 4 novembre 2015 .

DOI : $10.7202 / 1063100 a r$

Perrenoud (Philippe). 2005. Métier d'élève et sens du travail scolaire. Paris : ESF.

Pringle (Emily). 2008. " Artists' perspectives on art practice and pedagogy », p. 41-50 in Creative Learning / sous la direction de Sefton-Green (Julian). Londres : Arts Council.

Pronovost (Gilles). 2002. Les Enquêtes de participation culturelle : Une comparaison FranceQuébec-États-Unis. Québec : Institut de la statistique du Québec.

Rancière (Jacques). 1987. Le Mâtre ignorant : Cinq leçons sur l'émancipation intellectuelle. Paris : Fayard.

Rancière (Jacques). 2009. Et tant pis pour les gens fatigués : Entretiens. Paris : Éditions Amsterdam.

Valentin (Élisa). 2006. L'Éducation aux arts et à la culture dans une perspective internationale : Un aperçu de quelques politiques nationales et territoriales et des principaux impacts relevés dans la littérature. Québec : INRS.

\section{Notes}

1 Le terme " projet artistique » désigne ici un dispositif qui permet de décliner le travail éducatif dans les écoles en fonction d'un projet culturel à caractère artistique (arts visuels, musique, littérature, danse, théâtre, etc.) en partenariat avec un organisme culturel et un artiste.

2 Nathalie Heinich (2005) appelle « régime de singularité » le mouvement qui se met en place dans le domaine de l'art dans le courant du xixe siècle et qui a consisté à donner de la valeur au fait d'être original, hors du commun, et ne pas faire les choses comme les autres. Selon N. Heinich, le régime de singularité s'oppose au " régime de communauté » où l'artiste doit suivre les traditions et reproduire les conventions, sans toutefois le remplacer, car il existe toujours des règles, même si elles sont implicites, pour discriminer ce qui relève de l'art ou non. 
3 La liste des disciplines artistiques admissibles au programme Libres comme l'art comprend : les arts visuels, les arts numériques, le cinéma et la vidéo, la danse, la littérature, les métiers d'art, la musique, les nouvelles pratiques artistiques (interdisciplinarité et multidisciplinarité), les arts du cirque et le théâtre. L'ensemble des critères et normes peut être consulté sur le site du Conseil des arts de Montréal : https://www.artsmontreal.org/fr/programmes/libres-comme-lart.

4 L’activité d’idéation désigne les activités de conception d’idées (Bonnardel, 2009).

$5 \mathrm{Au}$ Québec, les élèves apprennent à inventer et créer des musiques dès le primaire, et à transcrire sur papier leurs idées en représentant graphiquement les éléments du langage musical utilisés à l'aide d'un code de notation personnel, traditionnel ou non traditionnel.

6 Dans Du spirituel dans l'art, V. Kandinsky (1989 : 109) définit par le terme de " nécessité intérieure » la nécessité pour l'artiste d'exprimer ce qui est propre à sa personne, à son époque et à l'art.

7 Il peut paraître exagéré de parler d' « œuvre professionnelle » dans le cadre d'une œuvre réalisée par des élèves avec des artistes, mais il s'agit ici d'une particularité des projets Libres comme l'art : l'artiste travaille avec les élèves sur son propre projet de création et non sur celui des élèves et, comme toute autre œuvre d'art, l'œuvre réalisée doit répondre aux critères artistiques attendus par le marché de l'art.

\section{Pour citer cet article}

Référence papier

Myriam Lemonchois, « La participation à des projets artistiques, vecteur d'émancipation ? Le cas de projets de création dans des écoles montréalaises en milieu défavorisé », Culture \& Musées, 26 | 2015, 159-175.

Référence électronique Myriam Lemonchois, « La participation à des projets artistiques, vecteur d'émancipation ? Le cas de projets de création dans des écoles montréalaises en milieu défavorisé », Culture \& Musées [En ligne], 26 | 2015, mis en ligne le 19 juin 2018, consulté le 16 juin 2021. URL : http://journals.openedition.org/culturemusees/386; DOI : https://doi.org/10.4000/culturemusees.386

\section{Auteur}

\section{Myriam Lemonchois}

Myriam Lemonchois est professeure agrégée en didactique des arts à l'université de Montréal. Ses recherches portent sur les finalités et les modalités de l'éducation artistique dans le contexte de l'enseignement des arts et dans celui des projets artistiques et culturels, en particulier ceux qui permettent aux artistes de développer des projets de création avec des élèves dans des écoles primaires ou secondaires. Dernier ouvrage paru : Pour une éducation esthétique : Discernement et formation de la sensibilité, 2002. Paris : L'Harmattan.

Courriel : myriam.lemonchois@umontreal.ca

\section{Droits d'auteur}

Culture \& Musées 